\title{
MR CLEAN-NO IV: Intravenous treatment followed by intra-arterial treatment versus direct intra-arterial treatment for acute ischemic stroke caused by a proximal intracranial occlusion.
}

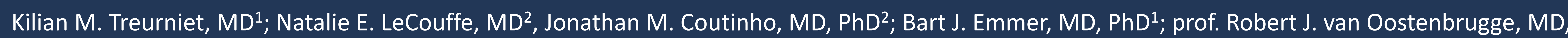

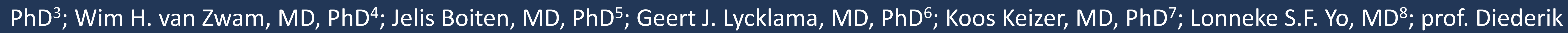
W.J. Dippel, MD, PhD'; Adriaan C.G.M. van Es, MD, PhD1 ${ }^{10}$; prof. Charles B.L.M. Majoie, MD, PhD¹; prof. Yvo B.W.E.M. Roos, MD, PhD²

From the ${ }^{1}$ department of radiology and nuclear medicine, Academic Medical Center Amsterdam, the Netherlands; ${ }^{2}$ department of neurology, Academic Medical Center, Amsterdam, the Netherlands ${ }^{3}$; department of neurology, MUMC, Maastricht, the Netherlands; ${ }^{4}$ department of radiology, MUMC, Maastricht, the Netherlands; ${ }^{5}$ department of neurology, Haaglanden Medisch Centrum, the Hague, the Netherlands; ${ }^{6} \mathrm{department} \mathrm{of} \mathrm{radiology,}$ Haaglanden Medisch Centrum, the Hague, the Netherlands; ${ }^{7}$ department of neurology, Catharina Hospital, Eindhoven, the Netherlands; ${ }^{8}$ department of radiology, Catharina Hospital, Eindhoven, the Netherlands; ${ }^{9}$ department of

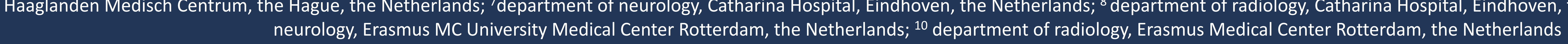

\section{RATIONALE}

- Rates of early recanalization with intravenous rtPA (IVT) in patients with acute ischemic stroke (AIS) with proximal intracranial occlusion are low

- Rates of symptomatic intracranial hemorrhage $(\mathrm{sICH})$ in the MR CLEAN trial were equal in both treatment groups (IVT vs IVT+intraarterial treatment [IAT]) $\rightarrow$ complication of IVT

- Direct IAT was at least as effective as IVT+IAT in patients who had a contraindication for IVT

\section{OBJECTIVES}

- Determine effect of direct IAT compared with IVT+IAT on functional outcome

- Non-inferiority of direct IAT relative to IVT+IAT on functional outcome

\section{STUDY DESIGN}

Prospective, randomized clinical trial with observer-blinded assessment of primary outcome (PROBE).

Patient flow in the study
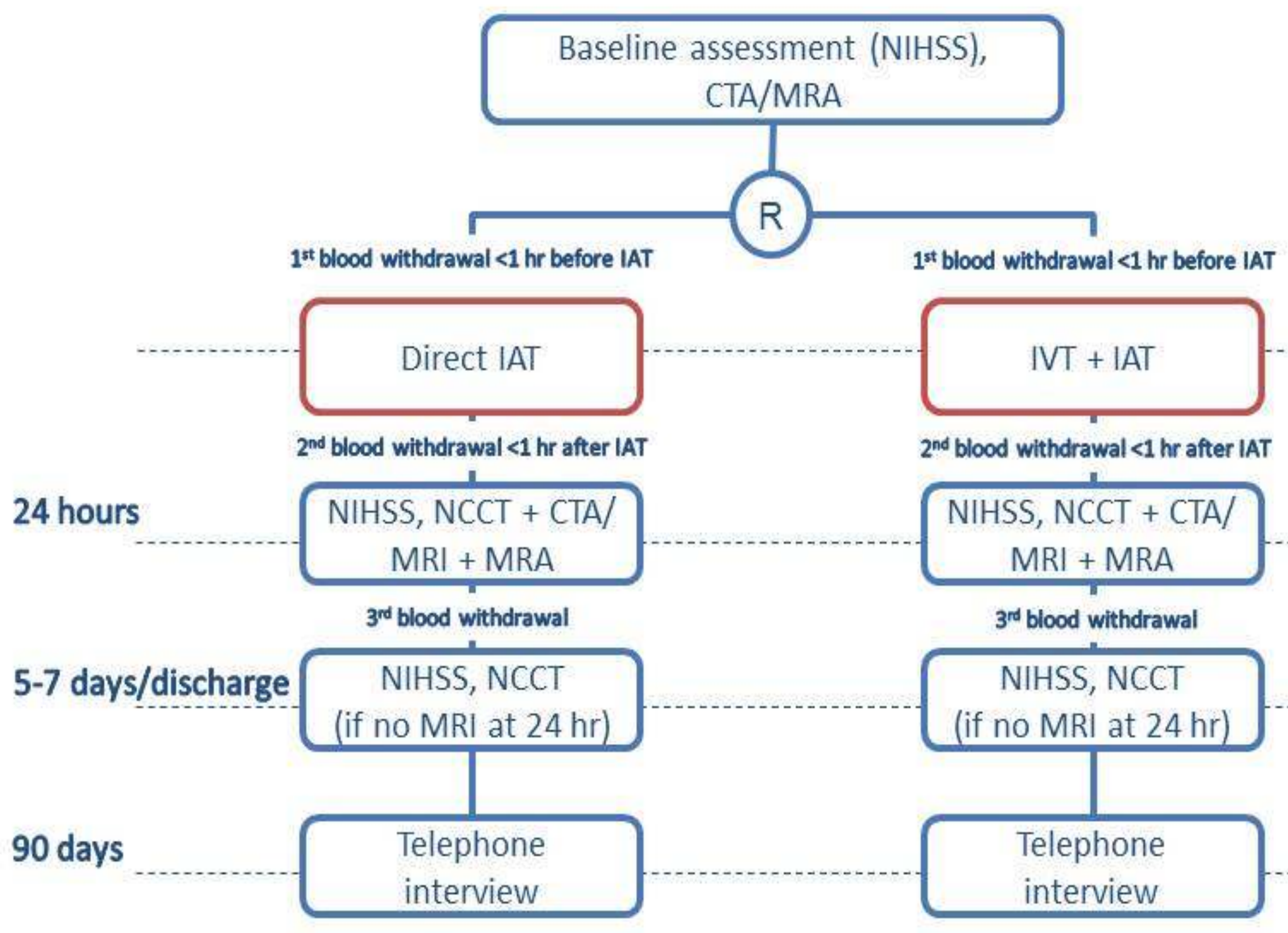

\section{PRIMARY OUTCOME}

Common Odds Ratio of modified Rankin Scale score at 3 months (mRS)

\section{SECONDARY / SAFETY OUTCOMES}

- Mortality at 90 days ( \pm 14 days)

- $\mathrm{SICH}$

- Extended treatment in cerebral ischemia (eTICI) score on final angiography of IAT

- Recanalization rate at 24 hours, assessed with CTA/MRA

- Score on the NIHSS at 24 hours and 5-7 days, or at discharge

- Final infarct volume at 5-7 days

STUDY POPULATION

Sample size: 540 patients.

Inclusion criteria

AIS

Proximal intracranial occlusion(CTA/MRA)

NIHSS $\geq 2$

Written deferred consent

\section{KEY STATISTICS}

- Netherlands: 2 participating centers, 15 planned to join

- Belgium: UZ Leuven planned to join

- France: Montpellier CHU planned to join

\section{TRIAL PROGRESS}

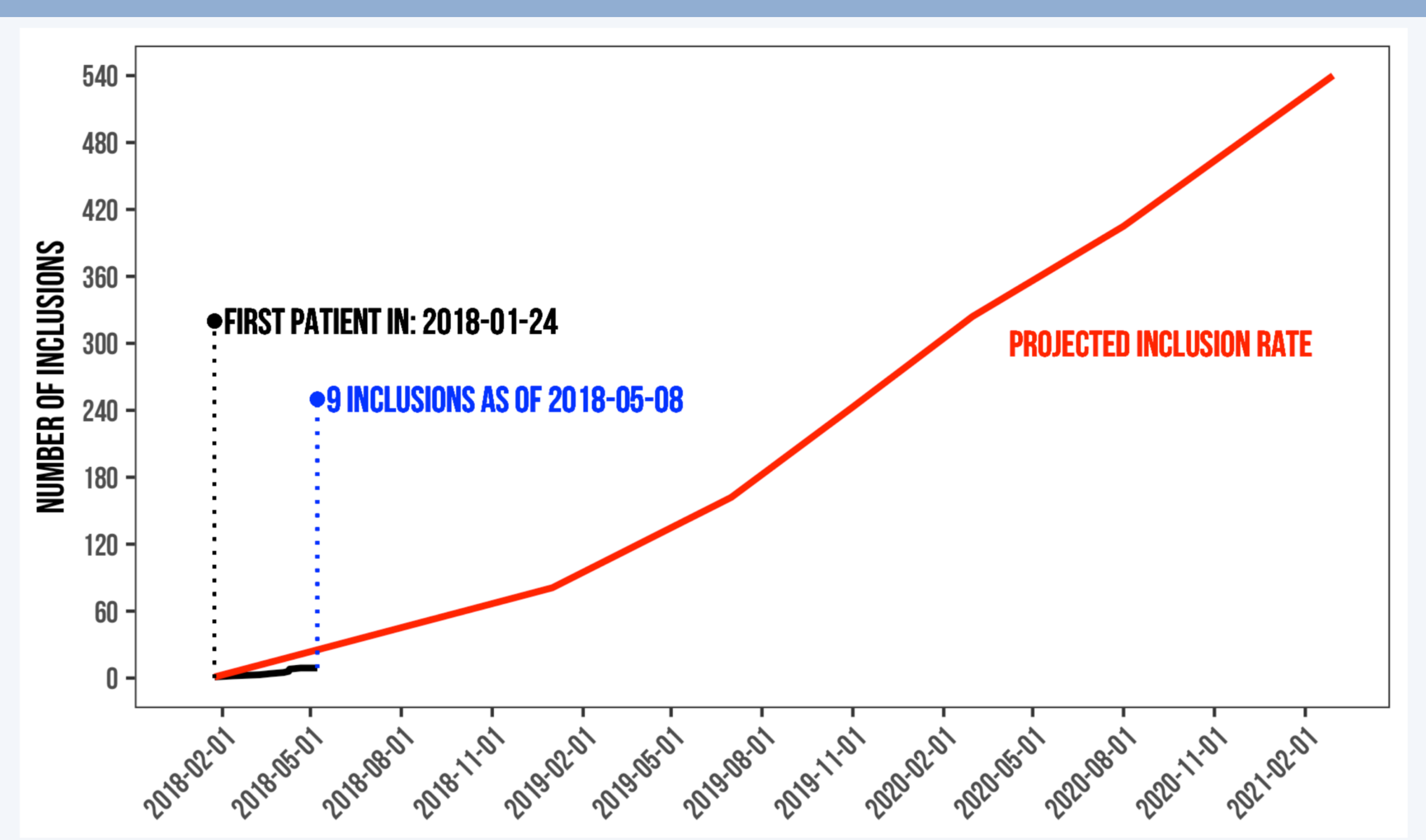

Natalie LeCouffe, MSc, PhD student | noiv-trialoffice@amc.uva.nl | www.mrclean-noiv.nl | ISRCTN80619088

$\Gamma$

FUNDED BY

(1) Hartstichting

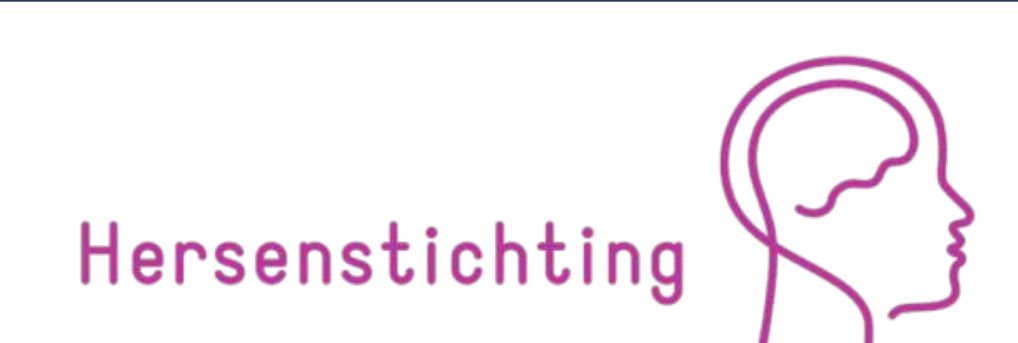

Hersenstichting

Stryker Penumbra P

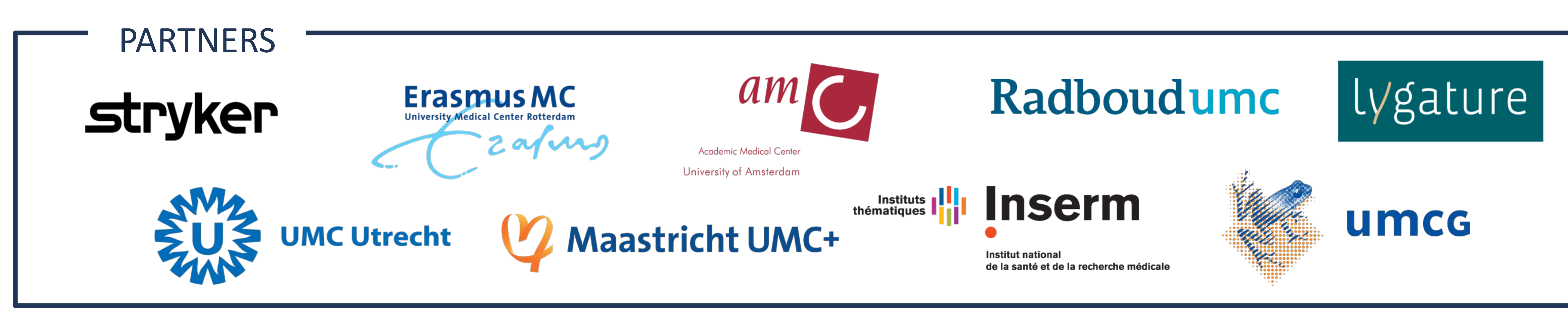

